\title{
Survey on Understanding Blockchain Technology and its Applications
}

\author{
K. Mayukha \\ Software Engineer
}

\begin{abstract}
The gap between the technology and critical infrastructure is shooting up rapidly. As the online revolution is surpassing the offline reality, a bold new approach to the prevailing system is demanded to mitigate the growing speculations. With emerging technologies such as IoT, AI, Cloud, Robotics, etc, Blockchain Technology will conclusively become a fundamental pillar in driving Industry 4.0. With IoT as the new network and AI as the new brain, Blockchain Technology is a new link to revolution, which represents a new way of storing data and simultaneously performing immutable and secure transactions than the current internet.
\end{abstract}

\section{Keywords}

Blockchain, Smart Contracts, Decentralization, Immutability, Security, Cryptography, Transparency, Trust

\section{INTRODUCTION}

The Blockchain is a decentralized ledger, or a list, of all transactions across a peer-to-peer network. This is the technology underlying Bitcoin and other cryptocurrencies, and it has the promising capability to revise a wide variety of business processes. Using this technology, the participants in the network can transfer value across the internet without requiring a central third party. Consequently, the buyer and seller can interact directly without demanding verification by a trusted third-party intermediary. Transactions are not anonymous, but they are pseudonymous: a transaction record is created, but identifying information is encrypted, and no personal information is shared.

If the internet is the foundation for digital innovation of all kinds, Blockchain technology is the underpinning of a radical rethinking ofhow the things are paid - as well as how it is verified, who owns what and who has the right to buy and sell it [1].

The usage of Blockchain is increasing significantly as it is more secure and because of many of its high attributes.

The below graph depicts the surge in the market value for Blockchain applications.

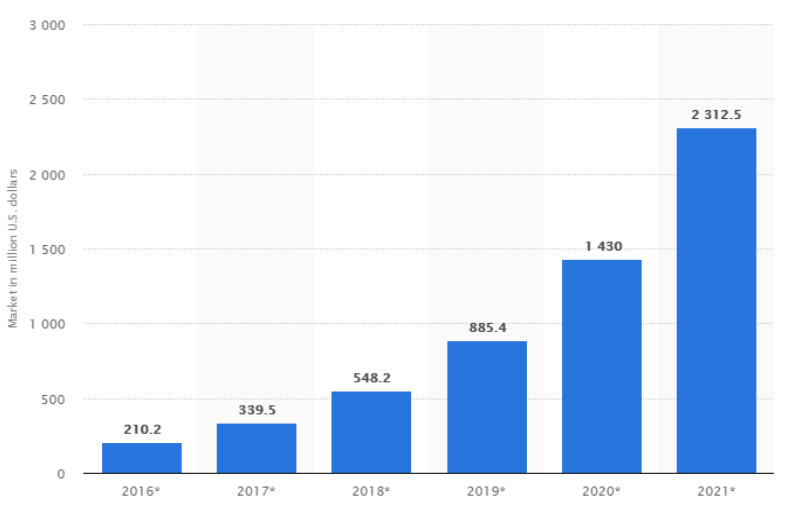

Figure1: Global Market for Blockchain technology

\section{KEY FEATURES OF BLOCKCHAIN}

\subsection{Immutability}

Blockchain is an immutable record of transactions, i.e. once the block is appended to the Blockchain it cannot be changed. This feature assures that the data in the block is tamper-proof as no one can encroach into the system and alter the data. Therefore, this created trust in the transaction records.

\subsection{Consensus}

Blockchain keeps the ledger transactions synchronized across the network to ensure that ledgers only update when the appropriate participants approve transactions. This process is called consensus. There are various consensus mechanism algorithms followed such as proof of work (POW), proof of stake (POS), delegated proof of stake (DPOS), etc. In this way, security is established in Blockchain.

\subsection{Decentralized}

Blockchain is independent of the central authority as the network is maintained by a group of nodes. The transactions are peer-to-peer without a third party involvement, by which there are zero scams or fraudulent activities.

\subsection{Transparent}

Transparency is one of the important characteristics of Blockchain. As Blockchain is an open ledger, anyone can access and audit the transactions. Every change on the Blockchain is evident and makes it more tangible.

\section{How Does BlockchainWorks?}

3.1 Either one of the nodes across the Blockchain network can start a transaction by digitally signing with their unique private key which is created via cryptography. A transaction can constitute many activities such as transfer of assets between the users on the Blockchain network, uploading the user data, etc.

3.2This transaction is broadcasted for the peers to validate. The validation is done based on the consensus algorithms which are the fixed norms used to reach a common agreement among the peers in the network.

3.3Once the transaction is validated, a block is created to represent the transaction containing- hash i.e. a value returned by the hash functions used such as SHA3-256 and Keccak256 , source and destination addresses, hash pointer of the previous block, and other valid information. Now the transaction is said to be approved.

3.4The block is then added to the Chain which provides a permanent, non repudiable and transparent record of the Blockchain.

3.5These blocks are added in a chronological order, in a way that resembles a chain, hence the name Blockchain [2] 


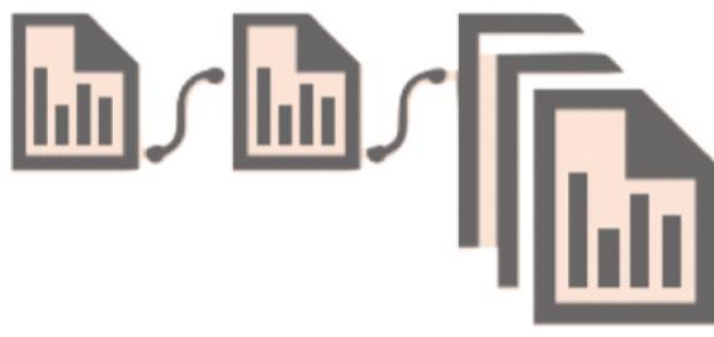

Figure2: Formation of Blockchain

\section{APPLICATIONS OF BLOCKCHAIN}

In situations where trust levels are low, due to differences in operational and regulatorylandscapes, such as multi party cross border transactions, the transparency offered by Blockchain could help by making the lack of trust, less of a hurdle, in these interactions. With its ability to forge more fairness, resilience and efficiency, the technology is influencing different sectors in a broad spectrum.

\subsection{In mitigating cyber-attacks:}

On the report of identity theft expert Life lock, more than 16 million. Americans complained of identity fraud and theft in 2017 alone, with an identity being stolen every two seconds [3]. By storing personal identity information such as birth certificates, social security numbers, debit/credit card details and other sensitive data on a decentralized Blockchain ledger, integrity and confidentiality of the data is maintained and also the government could see a drastic descend in identity theft claims and other cyberattacks. This decentralized form of data storage makes it harder for the hackers to penetrate the data storage systems, hence the data is protected.

\subsection{DeFi (or) Decentralized Finance:}

The financial services segment dominated the market in 2020 and accounted for more that $38.0 \%$ share of the global revenue, as shown. [4]

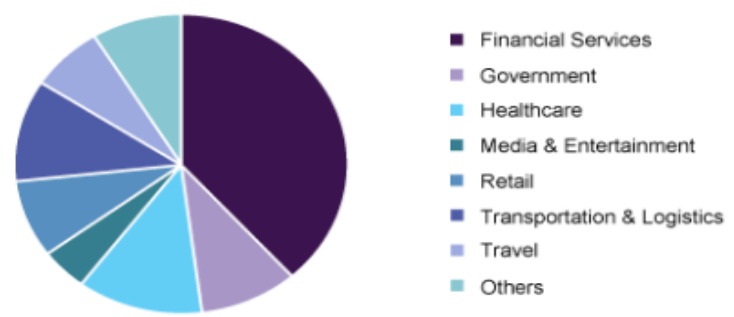

Figure3: Blockchain Market Share in 2020

In 2008, the world witnessed the great recession, also called Global Financial Crisis [5], which was caused by dysregulation in the financial industry such as bankruptcy of the iconic US investment bank, the Lehman Brothers Holdings. Owing to this violation of people's trust by central banks and other banks, this economic havoc called for a transition. A transition in the financial province. After 2 months of this crisis, bitcoin was proposed, introducing the world of finance to a simple yet powerful concept of decentralization through Blockchain. DeFi is referred to as financial applications which are developed using Blockchain technology, thus disrupting the traditional finance world. DeFi comprises applications and protocols which are transparent and peer-to-peer developed on decentralized Blockchain networks, such as Cross Border Transactions, Capital Markets, Trade Finance etc, that require no access rights for easy lending, burrowing, or trading of financial appliances.

\subsection{Blockchain in Health Care:}

Healthcare is a data-intensive clinical domain where a huge amount of data are generated, accessed, and disseminated on a regular basis. Storing and disseminating this large amount of data is crucial, as well as significantly challenging, due to the sensitive nature of data and limiting factors, such as security and privacy [6]. In such a scenario, Blockchain technology comes into the picture where the medical records are stored in a decentralized network in which immutability is maintained by the peer-to-peer network of computers, thus protecting the security and privacy of the data. A range of issues including data privacy, data integrity, data sharing, record keeping, patient enrolling [7], and so on, may arise in clinical trials. Blockchain, being the next internet generation [8], can provide viable solutions to these problems.

\subsection{Internet of Things Use Cases:}

The Internet of Things (IoT) is the next looming technology in Blockchainapplications. With an estimated 8.4 billion IoT devices being connected day in day out, IoT applications have many safety concerns such as data theft, information breach etc. Hackers have thus far managed to disable cars remotely entirely, control implanted cardiac devices, even used a refrigerator to attack businesses and launch the world's most massive DDoS attack [9]. Blockchain-affiliated IoT adds a higher level of security in preventing data breaches by making use of Blockchain's core functionalities - transparency, immutability and decentralization. Blockchain consists of the most robust encryption standards which establishes a much needed layer of security in the IoT stack. Any hacker or a malignant object will have to bypass this high-level security layer to access IoT devices, which makes cyber-attacks a way more problematic and time-consuming.

\subsection{Non-Fungible Tokens (NFTS):}

Non-Fungible Tokens (NFTs) [10] have been the trending Blockchain application since cryptocurrency. 2021 brought a huge advancement in these digital items that are currently taking the world by storm. NFTs are unique digital tokens such as art, music, image or videos, GIFs etc. which are sold on the Blockchain, ensuring that the full rights of the token are claimed by the sole owner. One of the memorable GIF, 2011 meme Nyan Cat, was sold for $\$ 600,000$ in ethereum on the blockchain [11]. In March 2020, the digital artist "Beeple", sold his digital work The First 5000 Days for $\$ 69$ million [12]. Through NFTs the buyers get a fortune to own digital moments, art and culture that prevails us.

\section{ACKNOWLEDGEMENTS}

I express my sincere gratitude towards my parents for their constant support and also I would like to thanks my mentor Mr. Vivekanandhan Muthulingam for his right through guidance

\section{CONCLUSION}

Now there is a technology for trust. With political will, investment and industry collaboration, it is believed that Blockchains can enhance the way business is done. Blockchain addresses a wide set of business challenges, by taking out cost, improving efficiencies and increasing accessibility across every industry. Not only in business, but Blockchain is spreading far beyond the financial world. With many practical applications, Blockchain is making a mark in every fibre ofindustry. So it's high time to embrace the technology and 
credit the paradigm shift by making the current systems more accurate, secure, efficient and cost-effective with fewer middlemen.

As part of the ongoing research, further analysis is being conducted on other consensus algorithms and hypotheses to determine how they can be integrated into blockchain applications in various sectors.

\section{REFERENCES}

\section{[1] $\mathrm{PwC}$}

FinTech

Q\&A

https://www.pwc.com/gr/en/publications/assets/qa-what-isblockchain.pdf

[2] Understanding Blockchain Technology means for Your Business https://www.smefinanceforum.org/sites/default/files/post/files/ 160225_insights_blockchain_0.pdf

[3] How common is identity theft https://www.lifelock.com/learnidentity-theft-resources-how-common-is-identity-theft.html

[4] Progression of Blockchain Technology Market Size https://www.grandviewresearch.com/industryanalysis/blockchain-technology-market

[5] Global Financial

Crisis https://en.wikipedia.org/wiki/Financial_crisis_of_2007\%E2\% $80 \% 932008$

[6] Griebel, L.; Prokosch, H.U.; Köpcke, F.; Toddenroth, D.;
Christoph, J.; Leb, I.; Engel, I.; Sedlmayr, M. A scoping review of cloud computing in healthcare. BMC Med. Inform. Decis. Mak. 2015, 15, 17. https://pubmed.ncbi.nlm.nih.gov/25888747/

[7] How Blockchain Will Revolutionise Clinical Trials, June $2018 . \quad$ https://pharmaphorum.com/views-andanalysis/how-blockchain-will-revolutionise-clinical-trialsclinical-trials/

[8] Moe Alsumidaie, Blockchain Concepts Emerge in Clinical Trials, Applied Clinical Trials, May 2018.https://www.appliedclinicaltrialsonline.com/view/bl ockchain-concepts-emerge-clinical-trials

[9] Benefits of Blockchain in IoT https://www.geospatialworld.net/blogs/benef its-ofblockchain-in-iot/

[10] A beginner's guide to NFTS for cryptoart https://builtin.com/blockchain/nft-cryptoart-guide

[11] 'Nyan Cat' flying Pop-Tart meme sells for nearly $\$ 600,000$ as one-of-a-kind cryptoart https://www.businessinsider.in/tech/news/nyan-catflying-pop-tart-meme-sells-for-nearly-600000-as-one-ofa-kind-crypto-art/articleshow/81179350.cms

[12] Beeple sold an NFT for \$69 million https://www.theverge.com/2021/3/11/22325054/beeplechristies-nft-sale-cost-everydays-69-million 